\title{
AVALIAÇÃo DOS PROGRAMAS DE PÓS-GRADUAÇÃO EM QUÍMICA NO BRASIL: VERSÃo 2002
}

\author{
Alfredo Arnóbio S. da Gama* \\ Departamento de Química Fundamental, Universidade Federal de Pernambuco, Av. Prof. Moraes Rego, 1235, 50670-901 Recife - PE \\ Solange Cadore \\ Instituto de Química, Universidade Estadual de Campinas, CP 6154, 13084-971 Campinas - SP \\ Vitor F. Ferreira \\ Departamento de Química Orgânica, Instituto de Química, Universidade Federal Fluminense, Outeiro de S. João Batista, s/n, \\ 24020-150 Niterói - RJ
}

Recebido em 2/4/03

\begin{abstract}
EVALUATION OF GRADUATE PROGRAMS IN CHEMISTRY IN BRAZIL: 2002 VERSION. The graduate programs in Chemistry were evaluated and classified from the data collected for the period 1998-2000 by CAPES, the Minister of Education agency that is responsible for the regulation and support of this level of human resources formation. The next classification will be made in 2004, from the data of the period 2001-2003. The years in between, like the present year, are used for an evaluation without classification, from what comments are emitted to the programs to know how their data are improving or not, before the new ranking reporting. The progresses in the area of chemistry in Brazil are analysed from the confrontation of the present data with that of all previous evaluations, since 1983. The results confirm that the research and graduate formation in the area of Chemistry is continuously improving in Brazil, particularly during the last seven years.
\end{abstract}

Keywords: graduate programs in chemistry; graduate programs in Brazil; chemistry in Brazil.

\section{INTRODUÇÃO}

A CAPES foi fundada em 1951 como uma Campanha para o Aperfeiçoamento de Pessoal Docente para o Ensino Superior e, desde então, vem progressivamente ampliando suas ações e permanentemente atuando no gerenciamento, credenciamento e normalização da pósgraduação stricto sensu ${ }^{1}$. No cumprimento de sua missão, a CAPES desenvolveu um sistema de avaliação da pós-graduação, que pode ser considerado uma das mais bem sucedidas iniciativas da educação superior brasileira ${ }^{2-4}$. Este sistema de avaliação foi construído com a participação da comunidade acadêmica e vem sendo continuamente aperfeiçoado de maneira a se tornar mais confiável e transparente ${ }^{5,6}$, utilizando, entre outras ferramentas, o acompanhamento e avaliação do processo por membros da comunidade científica internacional, a disponibilização dos dados fornecidos por todos os programas para consulta e análise, por qualquer pessoa, através da sua página na internet e uma participação cada vez maior do Conselho Técnico Científico (CTC) no acompanhamento dos critérios de avaliação das áreas e consistência dos conceitos atribuídos. Ao fim de cada processo de avaliação são discutidos os procedimentos e resultados, procurando identificar os aspectos que podem ser melhorados, com base nos questionamentos e sugestões dos docentes que se envolveram no processo, tanto como avaliadores quanto como avaliados. As manifestações dos programas são fontes valiosas para o aperfeiçoamento contínuo da avaliação da pós-graduação brasileira.

Particularmente, as exigências quanto à produção intelectual, em relação à quantidade, sua distribuição entre os docentes do Programa e qualidade dos veículos de divulgação, além da co-autoria com discentes do programa, representam um importante indutor do desenvolvimento da pesquisa e inserção internacional. Os indicadores

*e-mail: arnobio@npd.ufpe.br de inserção internacional estão sendo discutidos em cada área e nas grandes áreas (que reúnem áreas afins). Caminha-se para um consenso quanto às publicações em periódicos internacionais de mais elevado índice de impacto ${ }^{7,8}$, embora os valores destes índices devam ser considerados dentro de cada área. Também concorda-se em considerar a participação de docentes no corpo editorial de periódicos internacionais, convites para palestras, cursos e avaliações no exterior, projetos de cooperação e recepção de alunos estrangeiros pelo programa.

A comissão de avaliação é composta por oito membros escolhidos pela CAPES, a partir de uma lista elaborada pela coordenação da área, e procura atender critérios de distribuição por subáreas da Química e por Regiões do País.

A recente avaliação da CAPES confirma que a pesquisa e a pósgraduação em Química permanecem em acelerada evolução. Os diversos indicadores de desempenho têm apresentado acentuado progresso ao longo dos últimos dezessete anos, conforme se pode constatar pela análise dos dados coletados no período 1983-2001.

A avaliação tem sido sempre feita pelos pares, com base em critérios definidos e divulgados, por meio do documento de área, disponível na página da CAPES, e publicações na Química Nova ${ }^{9}$.

A última definição do perfil de excelência (perfil do curso A) na área foi amplamente superada pela maioria dos cursos de Pós-Graduação em Química. Com a definição de uma nova escala (1 a 7) e critérios para promoção aos níveis superiores (6 e 7), era razoável esperar um crescimento ainda maior nos indicadores de desempenho dos programas, o que foi confirmado.

\section{CRITÉRIOS PARA AVALIAÇão DA ÁREA}

A avaliação dos programas de pós-graduação de todas as áreas se faz com base nos dados apresentados pelos programas no DATACAPES, os quais referem-se a: (1) corpo docente, (2) atividades de pesquisa, (3) atividades de ensino, (4) corpo discente, (5) 
teses e dissertações e (6) produção intelectual. A área tem liberdade para definir a ponderação dos itens que constituem o relatório.

$\mathrm{Na}$ área de Química, como nas demais áreas das Ciências Exatas e da Terra, valoriza-se mais a produção intelectual. Considera-se como tal os artigos completos publicados em periódicos indexados, ponderados em relação à classificação dos veículos, com base nos índices de impacto atribuídos pelo "Journal Citation Report (JCR)"10-12. Periódicos com índice de impacto igual ou superior a 1 são classificados como internacional A (IA), com índice de impacto inferior a 1 , mas igual ou superior a 0,5 , como internacional B (IB) e com índice de impacto menor que 0,5 , mas igual ou superior a 0,07 , como internacional C (IC). Periódicos com índice de impacto inferior a 0,07 ou que não aparecem no JCR são classificados como nacionais. Quando se trata de periódico novo, com corpo editorial altamente conceituado, a classificação é, em princípio, nacional A (NA). Porém, esta classificação pode ser alterada a partir da avaliação por membros da comunidade. Periódicos estrangeiros, com corpo editorial menos prestigioso são considerados como nacional B (NB). Finalmente, periódicos de circulação nacional e/ou reconhecidos em outras áreas do conhecimento, são classificados como nacional C (NC). O indicador produção intelectual representa $55 \%$ da avaliação dos programas de pós-graduação em Química. Além do quesito produção intelectual, este item reflete a qualidade e dedicação do corpo docente e o nível das atividades de pesquisa.

Outro indicador importante, o qual representou, na avaliação 1998-2000, 25\% da avaliação global, e que é utilizado para avaliar a qualidade das teses e dissertações e o corpo discente, é a participação dos alunos como co-autores nas publicações. Infelizmente, este indicador é sujeito a falhas, tendo em vista que o banco de dados da CAPES não considerou os alunos que concluíram o programa (egressos) e que têm os trabalhos com resultados de suas teses (dissertações) publicados em anos posteriores. Esta falha está sendo parcialmente corrigida nos dados de 2001, com a inclusão dos egressos de anos anteriores, a partir do entendimento da importância desta informação para a avaliação dos resultados da pós-graduação. O Comitê de Química está recomendando que os egressos ${ }^{13,14}$ sejam considerados, para efeito de participação discente nas publicações do programa, por um prazo de até três anos após a titulação.

O terceiro indicador, com peso de $20 \%$ na avaliação final, reflete a eficiência do programa na titulação de mestres e doutores, por meio de uma combinação do número de titulados por docente e do número de titulados por discente, além dos tempos médios de titulação. Este último indicador relaciona-se com os quesitos atividades de ensino, corpo discente e teses e dissertações.

O documento de área, elaborado ao final de cada processo de avaliação, e os critérios de avaliação da área, divulgados antes dos períodos de avaliação, estabelecem o perfil que os programas devem atender. De forma resumida, os programas de pós-graduação em Química devem procurar divulgar sua produção intelectual em veículos de circulação internacional de elevado índice de impacto, com boa participação dos alunos em co-autoria, tendo como base os resultados de suas teses e dissertações, as quais recomenda-se que sejam concluídas dentro dos prazos recomendados pela CAPES.

Mais adiante são apresentados os indicadores de desempenho dos programas de pós-graduação em Química, de acordo com os dados coletados para a avaliação no período 1998-2000 e 2001 comparando-os com os anos anteriores.

\section{METODOLOGIA}

$\mathrm{Na}$ análise dos indicadores de qualidade dos programas somente são considerados os dados fornecidos pelas respectivas coordenações e enviados à CAPES pelas Pró-Reitorias de Pós-Graduação das instituições por meio do aplicativo COLETA, desenvolvido para esse fim. A CAPES tem investido continuamente no aperfeiçoamento da coleta e tratamento dos dados enviados, disponibilizando para os avaliadores uma grande quantidade de informações. O comitê de avaliação na área de Química prioriza algumas informações, conforme já mencionado, verificando cuidadosamente a consistência das mesmas, tendo inevitavelmente que efetuar o levantamento manual de algumas delas, particularmente a mais importante, a produção científica em periódicos. Embora os periódicos tenham sido cadastrados e qualificados, falhas operacionais têm conduzido ao não reconhecimento de vários periódicos, seja por falta de uniformidade no registro destes, seja pela maneira como o registro pode ser feito.

Em relação ao corpo docente, considerou-se a qualificação, dedicação ao programa, contribuição para a produção e formação de recursos humanos, além da participação no ensino de graduação e orientação de iniciação científica.

Nas atividades de pesquisa, levou-se em conta as linhas de pesquisa e os projetos concluídos e em andamento, além da coerência entre estes e a produção do programa no período. Nas atividades de formação observou-se a estrutura curricular e a oferta de disciplinas.

$\mathrm{O}$ aspecto corpo discente inclui a titulação de mestres e doutores, os tempos médios de titulação e a relação entre as teses e as publicações, destacando-se particularmente a participação dos alunos como autores dos trabalhos publicados. A análise qualitativa e quantitativa da produção discente, além de considerar a relação entre as teses produzidas e as publicações, considera também o número de teses e dissertações em relação ao total de alunos e ao total de docentes do programa.

$\mathrm{O}$ item de maior peso, a produção intelectual, considera os trabalhos efetivamente publicados no período, em periódicos indexados e de circulação internacional, ponderados de acordo com os seus índices de impacto.

Relativo ao período 1998-2000, os cursos foram analisados inicialmente pelos critérios que definem um curso de excelência (perfil dos cursos A) e assim classificados entre 1 e 5 . Em seguida foram considerados outros critérios para diferenciação entre os cursos melhor classificados, verificando-se quais poderiam ser promovidos ao nível 6 e, a seguir, ao nível 7. Neste sentido considerou-se, como aspectos positivos, a presença de docentes com bolsa de produtividade do CNPq e liderança em seus campos de atuação, revelada pela participação em corpo editorial de periódicos, comitês e sociedades científicas, o histórico evolutivo do programa e a tradição na formação de recursos humanos.

\section{ANÁLISE DOS INDICADORES}

Para compreender melhor o desempenho e o desenvolvimento dos programas de pós-graduação em Química no país, é interessante considerar a evolução dos indicadores normalmente utilizados na análise dos cursos/programas de pós-graduação, cobrindo o período 1983-2001, a qual pode ser observada na Tabela 1 (dados gerais e consolidados). Os dados dispensam maiores comentários, tendo em vista que, em praticamente todos os casos, mostram uma evolução significativa, em termos qualitativos e quantitativos, da pós-graduação em Química no Brasil. Os dados entre 1983-1995 foram obtidos de Brocksom e Andrade ${ }^{15,16}$, exceto os dados de publicações do período 1994/1995, que foram extraídos do banco de dados da CAPES. Como o número de total de docentes neste período não foi informado, o mesmo foi estimado com base na média dos períodos anteriores. Os dados do período 1996/1997 foram obtidos de da Gama, Nome e Machado ${ }^{17}$. Neste período, a falta de distinção clara entre docentes permanentes e participantes dos programas pode ter induzido uma redução no cadastramento destes últimos. 
Tabela 1. Evolução dos indicadores normalmente utilizados na análise dos Cursos/Programas de Pós-Graduação entre 1983-2001

\begin{tabular}{|c|c|c|c|c|c|c|c|c|c|}
\hline Períodos & $83 / 84$ & $85 / 86$ & $87-89$ & $90 / 91$ & $92 / 93$ & $94 / 95$ & $96 / 97$ & $98-00$ & 2001 \\
\hline Total de docentes & 515 & 564 & 705 & 902 & 910 & 938 & 842 & 910 & 922 \\
\hline Publicações nacionais & 119 & 152 & 368 & 226 & 276 & 299 & 399 & 628 & 169 \\
\hline Publicações internacionais & 358 & 427 & 830 & 610 & 1090 & 1024 & 1870 & 4174 & 1707 \\
\hline Total de publicações & 477 & 579 & 1198 & 836 & 1366 & 1323 & 2269 & 4802 & 1876 \\
\hline Publicações/doc./ano (intern.) & 0,23 & 0,25 & 0,39 & 0,23 & 0,4 & 0,36 & 0,74 & 1,53 & 1,85 \\
\hline Publicações/doc./ano (total) & 0,46 & 0,51 & 0,85 & 0,46 & 0,75 & 0,71 & 1,35 & 1,76 & 2,03 \\
\hline Total de alunos & 875 & 1149 & 1660 & 2018 & 2347 & 2059 & 2981 & 3208 & 3535 \\
\hline Mestres titulados & 226 & 212 & 411 & 476 & 457 & 446 & 664 & 1124 & 419 \\
\hline Doutores titulados & 75 & 81 & 109 & 157 & 178 & 279 & 380 & 631 & 292 \\
\hline $\mathrm{T}_{\text {médio }}$ de titulação/mestrado (meses) & 48 & 51 & 46 & 46 & 43 & 42 & 38 & 33 & 30 \\
\hline $\mathrm{T}_{\text {médio }}$ de titulação/doutorado (meses) & 66 & 65 & 71 & 64 & 59 & 58 & 60 & 59 & 59 \\
\hline Alunos/docente & 1,7 & 2,04 & 2,35 & 2,24 & 2,58 & 2,2 & 3,54 & 3,53 & 3,83 \\
\hline
\end{tabular}

A Tabela 1 mostra claramente que os trabalhos produzidos pelos programas de pós-graduação em Química estão sendo cada vez mais publicados em periódicos indexados e de circulação internacional. No período entre 1983 e 2001 foram publicados 14.726 trabalhos, a maioria em revistas internacionais (82\%). A Figura 1 mostra que a relação entre as publicações em periódicos internacionais e nacionais tem aumentado significativamente nos últimos oito anos.

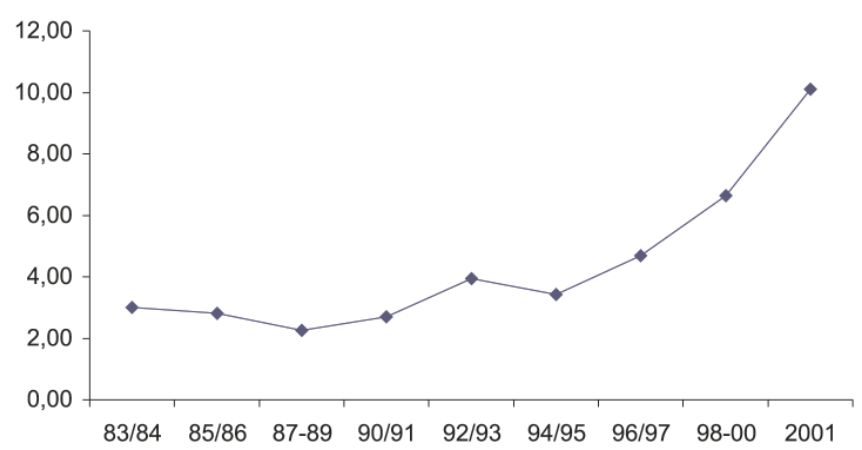

Figura 1. Relação entre publicações em periódicos internacionais e nacionais entre 1983 e 2001

Quando foi introduzida a classificação de periódicos não se encontrava nenhuma revista publicada no Brasil com índice de impacto superior a 0,5 , entretanto a comissão decidiu classificar o Journal of the Brazilian Chemical Society como IB, considerando a reconhecida margem de erro dos índices de impacto, particularmente para um periódico com as características deste ${ }^{18}$. O periódico Química Nova, que no período anterior havia sido classificado equivocadamente como nacional A, teve sua classificação corrigida para internacional $\mathrm{C}$. Isto não implicou nenhuma alteração na conceituação dos programas porque na primeira ocasião estabeleceu-se uma equivalência de valores entre os periódicos classificados como IC e NA. A Figura 2 mostra a distribuição da produção da pós-graduação em Química, de acordo com a classificação dos periódicos, evidenciando que $81 \%$ da produção científica da pós-graduação brasileira na área da Química está concentrada em revistas internacionais de nível A e B.

A produção científica da pós-graduação em Química mostra um crescimento significativo a partir do biênio 1992/1993, como pode ser visto na Figura 3.

Da mesma forma, o número de dissertações de mestrado defen-

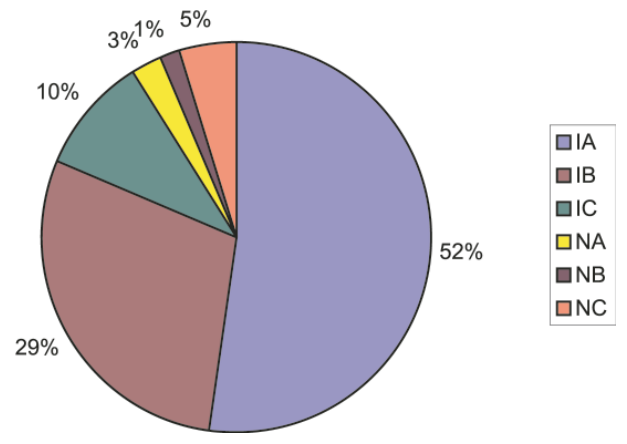

Figura 2. Distribuição dos artigos publicados em 2001, de acordo com o índice de impacto das revistas

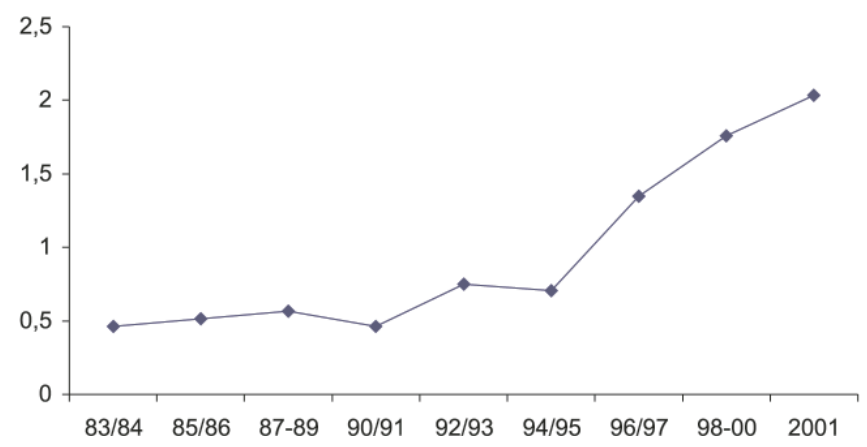

Figura 3. Variação do índice de publicações/docentelano (total), em função do período de análise, para o período 1983 a 2001

didas, que já passou por uma fase entre estacionário e decrescente, entre 1992 e 1995, apresenta-se em crescimento, assim como o número de teses de doutorado, como mostra o gráfico da Figura 4.

O tempo médio de titulação no mestrado apresentou uma diminuição a partir de 1987, comportamento esse que se manteve nos anos seguintes, acentuando-se no período de 1998-2000. Esse tempo ainda pode ser considerado elevado, uma vez que o recomendado pela Capes é de 24 meses. Por outro lado, o tempo médio de titulação no doutorado mantém-se constante desde 1994 e, aparentemente, é difícil descer do patamar de sessenta meses, conforme se observa na Figura 5. Aliás, este é o prazo máximo recomendado para aqueles que seguem diretamente para o doutorado, e que contribuem para o tempo médio calculado. 


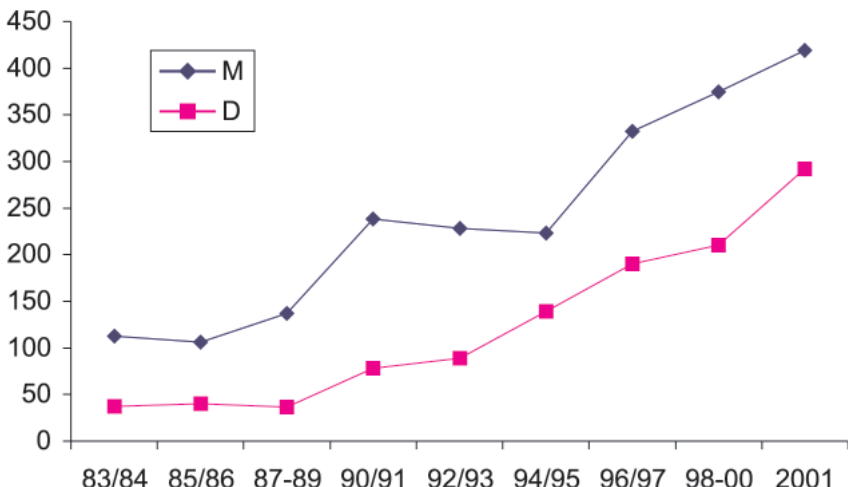

Figura 4. Média anual das dissertações de mestrado (M) e teses de doutorado (D) produzidas pelos programas de pós-graduação em Química, no período 1983-2001

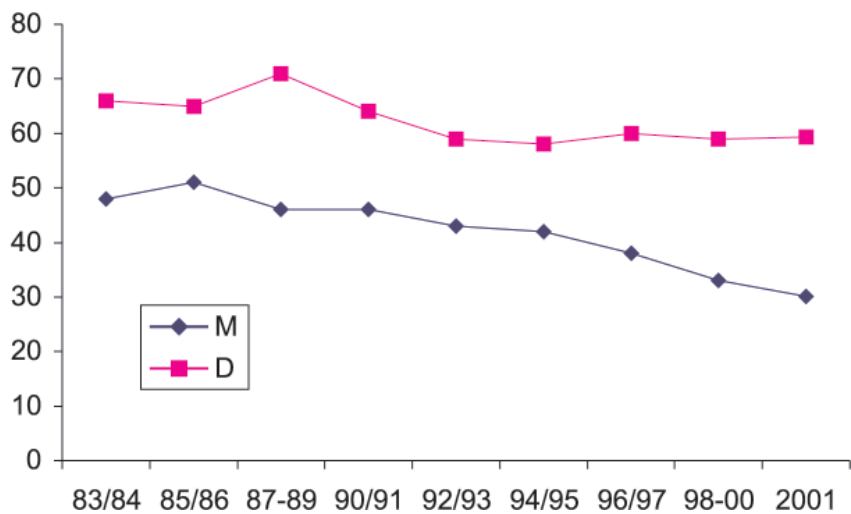

Figura 5. Evolução dos tempos médios de titulação no mestrado e no doutorado

Além de progredir qualitativamente, a pós-graduação em Química continua em crescimento. Não somente o número de alunos tem aumentado, mas também a oferta de programas nas diversas regiões do país. Como conseqüência da ampliação e consolidação dos programas ao nível de doutorado, tem sido significativo o número de pesquisadores qualificados disponibilizados anualmente para a sociedade. Com a baixa oferta de vagas para novos docentes nos centros mais tradicionais, começam a ser formados grupos de pesquisa mais fortes e dinâmicos em novos centros e, assim, surgem novos programas de pós-graduação.

\section{DISTRIBUIÇÃO REGIONAL DA PÓS-GRADUAÇÃO EM QUÍMICA}

Reconhecido o crescimento qualitativo e quantitativo da pós-graduação, e notadamente da produção científica brasileira, não apenas na área de Química, mas igualmente nas outras áreas das Ciências, evidencia-se a necessidade de avaliar as lacunas existentes e a distribuição regional. Recentemente estes assuntos foram tema de um seminário organizado pela CAPES e estão em evidência também em projetos da alçada do Ministério da Ciência e Tecnologia. Mais adiante comenta-se sobre algumas lacunas observadas na área da Química no Brasil. Nesta seção estão apresentados alguns indicadores qualitativos e quantitativos da distribuição regional da pós-graduação em Química.

Como seria de se esperar, a distribuição dos programas de pósgraduação em Química entre as diversas regiões do país reflete as disparidades econômicas e a política de investimento em Ciência e Tecnologia e incentivo à criação de programas de pós-graduação. A Figura 6 mostra que a maior concentração se dá nos três Estados da Região Sudeste, a mais desenvolvida do país.

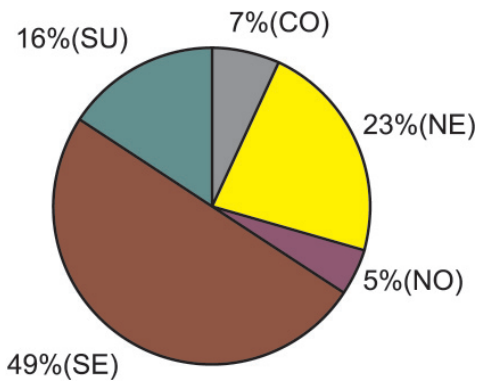

Figura 6. Distribuição regional da pós-graduação em Química

Há uma certa distorção destes dados devido à existência de programas divididos por subáreas da Química, predominantemente no Estado do Rio de Janeiro. Mesmo que os programas fossem unificados por Instituição, esta Região ainda manteria a hegemonia, embora bem mais reduzida (38\%), como mostra a Figura 7. Ainda assim, evidencia-se uma grande concentração, tendo em vista o reduzido número de Estados e o tamanho de alguns programas, notadamente o da UNICAMP, seguido pela UFMG e pela UFSCar. Fora da Região Sudeste, apenas na UFC existem programas de Química separados por subárea. Programas que inicialmente funcionavam separados na UNESP, na USP-RP e na USP foram unificados a partir de 1992, 1994 e 2002, respectivamente.

A Região Nordeste aparece em segundo lugar, em número de programas, mas estes distribuem-se entre oito Estados. O mais novo, em Sergipe, foi criado em 2002. Embora a Região Sul apareça na terceira posição, considerando o número de programas, todos os Estados contam com, pelo menos, um programa fora da Capital.

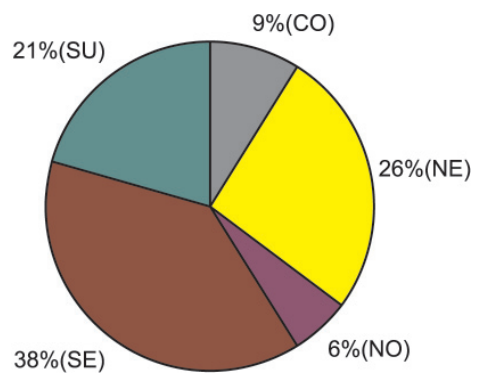

Figura 7. Distribuição regional da pós-graduação em Química unificando os programas por subárea em uma mesma instituição (exceto quando em campus distintos)

Quando se analisa a qualidade dos programas (Figura 8), a melhor média é também da Região Sudeste, mas as posições da Região Sul e Nordeste se invertem, o mesmo ocorrendo entre as Regiões Norte e Centro-Oeste, embora isto seja de pouco ou nenhum significado, tendo em vista o número reduzido de programas.

Quando se destaca a Região Sudeste e nela se analisa a distribuição da qualidade dos programas, de acordo com o conceito médio, nota-se um acentuado destaque para o Estado de São Paulo (Figura 9), embora quantitativamente haja uma maior concentração de programas no Estado do Rio de Janeiro. Novamente isto reflete as desigualdades econômicas, mas principalmente o investimento em pesquisa e pós-graduação, representado particularmente pela FAPESP. 


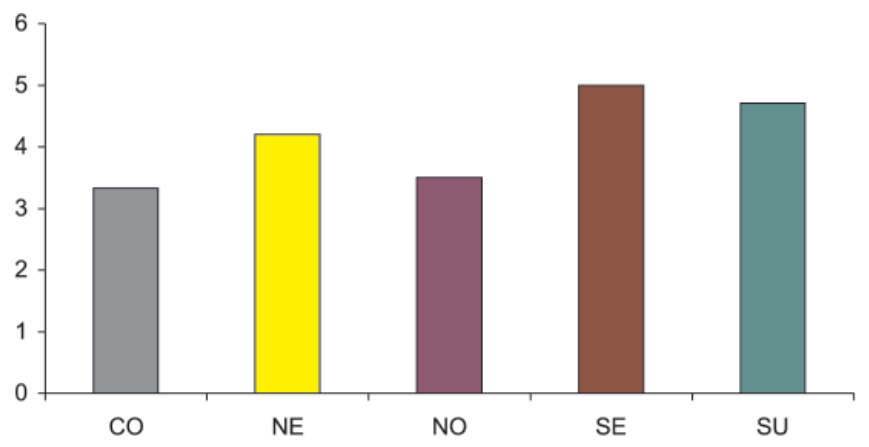

Figura 8. Conceito médio dos programas de pós-graduação em Química por Região

Nos últimos três anos a fundação carioca, FAPERJ, está mantendo um patamar de financiamento comparável ou mesmo superior à congênere paulista, considerando a dimensão da demanda qualificada, o que, se mantido para os próximos anos, poderá reduzir a disparidade atual, como sugerem os dados de 2001.

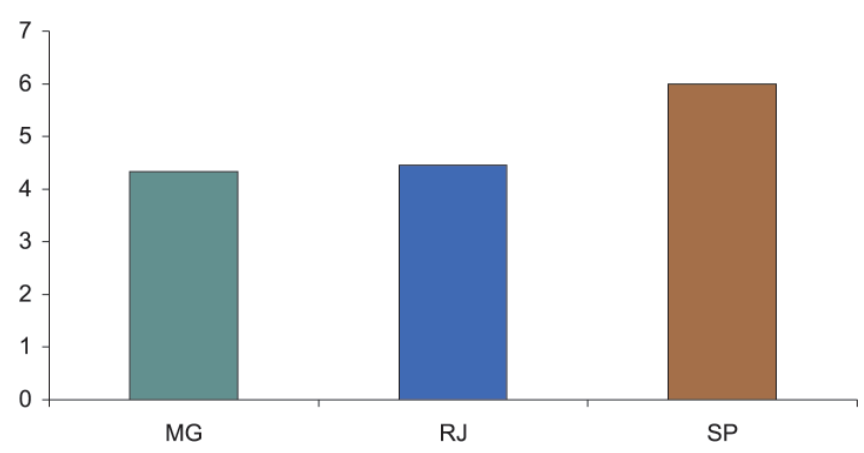

Figura 9. Conceito médio dos programas de pós-graduação em Química por Estado na Região Sudeste

Estas diferenças regionais ficam bem evidentes quando as Regiões são destacadas no quadro de distribuição dos conceitos dos programas, conforme mostrado na Figura 10.

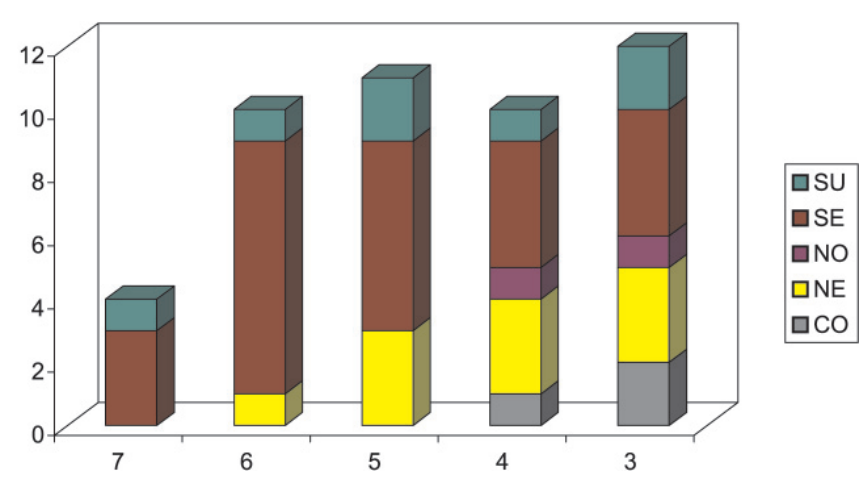

Figura 10. Distribuição dos conceitos da pós-graduação total e regional

Para que se mantenha um registro evolutivo individual dos programas de Química, apresenta-se a Tabela 2 onde estão indicados todos os conceitos dos programas de pós-graduação em Química entre 1980 e 2000.

\section{CONCLUSÕES}

Os dados coletados no período 1998-2000, a comparação com os períodos anteriores e uma primeira projeção com os dados de 2001 revelam, mais uma vez, um quadro positivo da pós-graduação em Química no país. Houve crescimento quantitativo tanto no número de alunos como na oferta de programas. A produção científica continua aumentando, mostrando ainda o crescimento do número de pesquisadores atuantes, conforme se observa pelo índice de trabalhos publicados por docente e ano. Crescem também os titulados em mestrado e doutorado. Merece especial atenção o crescimento no número de doutores formados, o qual tem sido responsável pela expansão do sistema de pesquisa e pós-graduação em Química no país, atingindo todas as regiões. São, hoje, 44 programas, 50\% com conceito de excelência (igual ou superior a 5) e $68 \%$ oferecendo o nível de doutorado. São titulados anualmente 210 doutores e 375 mestres. Cerca de 920 docentes estão publicando anualmente mais de 1870 trabalhos, dos quais 52\% em periódicos com índice de impacto 1 ou superior e $29 \%$ em periódicos com índice de impacto abaixo de 1 mas igual ou superior a 0,5 .

É notável observar que este crescimento, e também o bom desempenho da área, tem se verificado apesar da retração nos investimentos federais em pesquisa e desenvolvimento nos últimos anos. Até mesmo o número de bolsas para a pós-graduação, principalmente ao nível de mestrado, tem sofrido redução. É paradoxal, portanto, observar que está se produzindo recursos humanos em maior número e com melhor qualidade do que se fazia em períodos de maior investimento. Como a formação de recursos humanos é um processo demorado, os resultados somente aparecem após um período considerável, sendo o crescimento hoje observado justamente uma conseqüência destes investimentos, que impulsionaram o sistema até o estágio atual.

Vale a pena refletir sobre qual seria o futuro da pós-graduação em Química no Brasil se o contingente de doutores formados atualmente pudesse receber o mesmo volume de financiamento que era concedido tradicionalmente na década de setenta pelas diversas agências. A realidade é que vivencia-se uma crise sem precedentes no financiamento da pesquisa e pós-graduação no País. Porém, uma crise é ao mesmo tempo um momento de confronto e de novas oportunidades, onde a criatividade vem à tona buscando soluções com os recursos existentes. Por outro lado, a comunidade científica se une em busca de um objetivo comum, reivindicando uma política clara de financiamento à pesquisa.

Se os caminhos corretos forem trilhados, com a manutenção das taxas de bancada e/ou acadêmicas e a retomada de um programa de financiamento que permita a expansão do sistema de ciência e tecnologia, o futuro será altamente promissor. Trata-se de uma decisão política de fundamental importância, que não pode ser adiada, para assegurar o desenvolvimento integral da Nação.

Apesar de todo crescimento e progresso, ainda existem carências, que precisam ser supridas para dar continuidade à busca do estado da arte internacional na área de Química. Devem ser incentivadas pesquisas de ponta sobre a dinâmica de reações em feixes supersônicos, espectroscopia de alta resolução com laser ou radiação Síncroton, técnicas de análise instrumental, e instrumentação, com ressonância magnética, espectrometria de massas ou espectroscopia de alta resolução, química combinatória, entre outras. Além disso, ainda se exerce pouco a pesquisa nas interfaces, continuando-se a trabalhar a Química naquela divisão tradicional: Orgânica, Inorgânica, Analítica e Físico-Química. Não há uma interação efetiva nem mesmo com a Bioquímica ou outras áreas das ciências biológicas. No atual estágio de desenvolvimento dos programas de pós-graduação e da pesquisa em Química, talvez fosse mais interessante agrupar os pesquisado- 
Tabela 2. Evolução dos conceitos dos programas de pós-graduação em Química entre 1980 e 2000

\begin{tabular}{|c|c|c|c|c|c|c|c|c|c|c|c|c|c|c|c|c|c|c|c|c|c|c|}
\hline \multirow[t]{2}{*}{ Programa } & \multicolumn{2}{|c|}{ Início } & \multicolumn{2}{|c|}{80} & \multicolumn{2}{|c|}{81} & \multicolumn{2}{|c|}{82} & \multicolumn{2}{|c|}{$83 / 84$} & \multicolumn{2}{|c|}{$85 / 86$} & \multicolumn{2}{|c|}{$87-89$} & \multicolumn{2}{|c|}{ 90/91 } & \multicolumn{2}{|c|}{$92 / 93$} & \multicolumn{2}{|c|}{$94 / 95$} & \multirow{2}{*}{$\begin{array}{c}96 / 97 \\
M / D\end{array}$} & \multirow{2}{*}{$\begin{array}{l}98-00 \\
\mathrm{M} / \mathrm{D}\end{array}$} \\
\hline & $\mathrm{M}$ & $\mathrm{D}$ & M & $\mathrm{D}$ & M & $\mathrm{D}$ & M & $\mathrm{D}$ & M & $\mathrm{D}$ & M & $\mathrm{D}$ & $\mathrm{M}$ & $\mathrm{D}$ & $\mathrm{M}$ & $\mathrm{D}$ & $\mathrm{M}$ & $\mathrm{D}$ & $\mathrm{M}$ & $\mathrm{D}$ & & \\
\hline UA & 87 & & & & & & & & & & & & $\mathrm{C}$ & & C- & & C- & & $\mathrm{C}$ & & 3 & 3 \\
\hline UFPA & 87 & & & & & & & & & & & & $\mathrm{C}$ & & $\mathrm{C}$ & & C- & & $\mathrm{C}$ & & 3 & 4 \\
\hline UFMA & 95 & & & & & & & & & & & & & & & & & & $\mathrm{CN}$ & & 3 & 3 \\
\hline UFCE-QI & 77 & & $\mathrm{C}$ & & $\mathrm{C}$ & & $\mathrm{C}$ & & B & & B & & B & & $\mathrm{C}$ & & C & & $\mathrm{C}$ & & 4 & 5 \\
\hline UFCE-QO & 76 & 91 & B & & B & & B & & A & & A & & A & & A- & $\mathrm{SC}$ & A- & $\mathrm{CN}$ & B & $\mathrm{CN}$ & 4 & 4 \\
\hline UFRN & 90 & & & & & & & & & & & & & & $\mathrm{SC}$ & & $\mathrm{CN}$ & & $\mathrm{C}$ & & 3 & 4 \\
\hline UFPB & 86 & & & & & & & & & & $\mathrm{SC}$ & & $\mathrm{C}-$ & & $\mathrm{C}+$ & & $\mathrm{C}+$ & & B & & 4 & 5 \\
\hline UFPE & 89 & 89 & & & & & & & & & & & SA & SA & $\mathrm{C}$ & $\mathrm{C}$ & B & $\mathrm{CN}$ & A & $\mathrm{CN}$ & 6 & 6 \\
\hline UFAL & 92 & & & & & & & & & & & & & & & & $\mathrm{CN}$ & & $\mathrm{C}$ & & 4 & 4 \\
\hline UFBA & 68 & 92 & $\mathrm{C}$ & & $\mathrm{C}$ & & $\mathrm{C}+$ & & $\mathrm{C}+$ & & $\mathrm{C}$ & & B & & B & & B & $\mathrm{CN}$ & B & $\mathrm{CN}$ & 4 & 5 \\
\hline UFMG & 67 & 67 & A & A & A & A & A & A & A & A & A & A & A & A & A & A & A & $\mathrm{A}$ & A & $\mathrm{A}$ & 6 & 6 \\
\hline UFU & 98 & & & & & & & & & & & & & & & & & & & & 3 & 3 \\
\hline UFF-QO & 91 & & & & & & & & & & & & & & $\mathrm{SC}$ & & $\mathrm{CN}$ & & $\mathrm{C}$ & & 4 & 5 \\
\hline UFRRJ & 66 & 94 & $\mathrm{C}$ & & $\mathrm{C}$ & & B & & B & & $\mathrm{B}+$ & & A & & $\mathrm{A}$ & & A- & & B & $\mathrm{CN}$ & 4 & 5 \\
\hline UFRJ-QI & 93 & 93 & & & & & & & & & & & & & & & & & $\mathrm{CN}$ & $\mathrm{CN}$ & 4 & 4 \\
\hline UFRJ-QO & 63 & 63 & B & B & B & $\mathrm{C}$ & B & $\mathrm{C}$ & B & B & A & B & A & B & A & B & A & $\mathrm{B}+$ & $\mathrm{A}$ & B & 6 & 6 \\
\hline UFRJ-PN & 64 & 89 & A & & A & & A & & A & & A & & A & $\mathrm{SC}$ & A & B & A- & B & B & $\mathrm{C}$ & 5 & 5 \\
\hline UFRJ-CTP & 77 & 77 & B & $\mathrm{C}$ & B & $\mathrm{E}$ & B & $\mathrm{C}$ & $\mathrm{B}+$ & B & B & B & $\mathrm{B}$ & B- & $\mathrm{B}+$ & $\mathrm{B}$ & $\mathrm{B}+$ & B & A & A & 5 & 6 \\
\hline UFRJ-FQ & 72 & 91 & B & & $\mathrm{C}$ & & C- & & B & & B & & $\mathrm{B}+$ & & $\mathrm{B}+$ & SC & B & $\mathrm{CN}$ & B & $\mathrm{CN}$ & 5 & 5 \\
\hline PUC-RJ & 69 & 71 & B & $\mathrm{C}$ & A & B & A & A & A & A & A & A & $\mathrm{A}$ & A & $\mathrm{A}$ & $\mathrm{A}$ & A- & A- & B & B & 4 & 4 \\
\hline IME & 69 & 72 & A & B & B & $\mathrm{C}$ & B & $\mathrm{C}$ & B- & C- & B & $\mathrm{C}$ & B & B & B & B & $\mathrm{C}$ & $\mathrm{C}$ & $\mathrm{C}$ & $\mathrm{C}$ & 3 & 3 \\
\hline USP-FQ & 70 & 70 & A & A & A & A & A & A & A & A & $\mathrm{A}$ & A & A & A & A & A & A & A & $\mathrm{A}$ & A & 5 & 5 \\
\hline USP-QI & 70 & 70 & A & A & A & A & A & $\mathrm{A}$ & A & A & A & $\mathrm{A}$ & A & A & A & A & A & A & A & A & 5 & 6 \\
\hline USP-QO & 70 & 70 & $\mathrm{~A}$ & A & A & A & A & A & A & A & A & A & $\mathrm{A}$ & A & A & A & A & $\mathrm{A}$ & A & A & 6 & 6 \\
\hline USP-QA & 70 & 70 & A & A & A & A & A & $\mathrm{A}$ & A & A & $\mathrm{A}$ & A & A & $\mathrm{A}$ & $\mathrm{A}$ & A & A & $\mathrm{A}$ & A & A & 5 & 6 \\
\hline USP-SC-FQ & 71 & 76 & A & $\mathrm{A}$ & A & A & A & A & A & A- & A & A & $\mathrm{A}$ & A & A & A & A & A & A & $\mathrm{A}$ & 7 & 7 \\
\hline USP-SC-QA & 85 & 89 & & & & & & & & & $\mathrm{SC}$ & & $\mathrm{B}$ & & B & $\mathrm{C}$ & B & $\mathrm{C}$ & A & A & 6 & 5 \\
\hline UNESP-FQ & 85 & & & & & & & & & & $\mathrm{SC}$ & & SA & & B & & & & & & & \\
\hline UNESP-QI & 78 & 87 & $\mathrm{C}$ & & $\mathrm{C}$ & & B & & $\mathrm{B}$ & & B & & SA & SA & B & $\mathrm{C}+$ & & & & & & \\
\hline UNESP-QA & 85 & 85 & & & & & & & & & $\mathrm{SC}$ & $\mathrm{SC}$ & SA & SA & $\mathrm{B}$ & $\mathrm{C}+$ & & & & & & \\
\hline UNESP & & & & & & & & & & & & & & & & & $\mathrm{B}+$ & B & B & B & 5 & 6 \\
\hline UNICAMP & 72 & 72 & A & A & A & $\mathrm{A}$ & A & $\mathrm{A}$ & A & A & $\mathrm{A}$ & A & A & A & $\mathrm{A}$ & A & $\mathrm{A}$ & $\mathrm{A}$ & $\mathrm{A}$ & A & 5 & 7 \\
\hline USP-RP-FQ & 89 & & & & & & & & & & & & $\mathrm{SC}$ & & $\mathrm{C}$ & & $\mathrm{C}+$ & & & & & \\
\hline USP-RP-QI & 89 & & & & & & & & & & & & $\mathrm{SC}$ & & $\mathrm{C}$ & & B & & & & & \\
\hline USP-RP & & & & & & & & & & & & & & & & & & & $\mathrm{B}$ & B & 5 & 6 \\
\hline UFSCAR & 80 & 87 & $\mathrm{C}$ & & $\mathrm{C}$ & & B & & B & & B & & A & B & A & B & A & A & A & A & 6 & 7 \\
\hline UFPR & 91 & & & & & & & & & & & & & & & & $\mathrm{CN}$ & & $\mathrm{C}$ & & 4 & 5 \\
\hline UEM & 87 & & & & & & & & & & & & $\mathrm{C}$ & & $\mathrm{C}$ & & $\mathrm{C}$ & & $\mathrm{C}$ & & 3 & 4 \\
\hline UFSC & 71 & 88 & B & & B & & A & & A & & $\mathrm{A}$ & & $\mathrm{A}$ & $\mathrm{B}$ & $\mathrm{A}$ & B & A & $\mathrm{B}+$ & A & A & 6 & 7 \\
\hline UFRGS & 85 & 97 & & & & & & & & & $\mathrm{SC}$ & & $\mathrm{C}+$ & & $\mathrm{C}+$ & & $\mathrm{C}$ & & B & & 5 & 5 \\
\hline UFSM & 85 & 93 & & & & & & & & & $\mathrm{SC}$ & & $\mathrm{C}+$ & & $\mathrm{C}+$ & & B & $\mathrm{CN}$ & A & $\mathrm{CN}$ & 6 & 6 \\
\hline UNB & 74 & 91 & B & & B & & B & & $\mathrm{C}$ & & $\mathrm{B}$ & & $\mathrm{B}$ & & $\mathrm{B}$ & & B & $\mathrm{CN}$ & B & $\mathrm{CN}$ & 4 & 4 \\
\hline UFMS & 96 & & & & & & & & & & & & & & & & & & & & 3 & 3 \\
\hline UFG & 99 & & & & & & & & & & & & & & & & & & & & 3 & 3 \\
\hline UFPI & 99 & & & & & & & & & & & & & & & & & & & & & 3 \\
\hline UFJF & 00 & & & & & & & & & & & & & & & & & & & & & 4 \\
\hline
\end{tabular}

$\mathrm{SC}=$ sem conceito $\mathrm{CN}=$ curso novo $\mathrm{SA}=$ sem avaliação.

res que fazem síntese, os que usam ou desenvolvem equipamentos instrumentais e os que fazem modelagem ou simulação teórica. Em cada tema, entretanto, esses três tipos de químico devem trabalhar em estreita colaboração.

Finalmente, não se poderia deixar de destacar a necessidade da desconcentração regional e de combate à endogenia, revelada pelos $50 \%$ dos programas de pós-graduação no litoral e $49 \%$ na região sudeste. Como forma de ação para minimizar estas distorções na área, deve-se pensar na criação de facilidades regionais com equipamentos de grande porte, programas de bolsas (docentes e discentes) específicos, programas induzidos para a fixação de recém-doutores nas regiões menos desenvolvidas do país e facilitar a doação de equipamentos entre instituições do Brasil e do exterior. A área de Química não pode continuar a ignorar o interior do país.

Centrar esforços na direção de projetos envolvidos com inovação tecnológica, juntamente com formação de novas lideranças científicas com amplo leque de oportunidades na interface com outras áreas deve ser um desafio de todo o sistema de pós-graduação. A comunidade Química acadêmica precisa aprofundar a discussão dessas carências e elaborar projetos para superá-las em um futuro próximo. 


\section{AGRADECIMENTOS}

Os autores agradecem ao pessoal da CAPES, particularmente Rosana, Mônica, Sandra, Tereza e Cristiane, e aos colegas do Comitê, Faruk, Caetano, Douglas, Yoshitaka, Paulo Roberto, Mário, Luiz Carlos, Ícaro, Mangrich, Joel, Fabris e Zucco, que contribuíram para a coleta e análise dos dados apresentados.

\section{REFERÊNCIAS}

1. Resolução CNE/CES No 1 , de 3 de abril de 2001 estabelece as normas para o funcionamento de cursos de pós-graduação.

2. Martins, C. B. Em A Educação Superior no Brasil; Soares, M. S. A., ed.; CAPES-UNESCO, Brasília 2002.

3. Cagnin; M. A. H.; Quim. Nova 1994, 17, 258.

4. Barros, E. M. C.; Política de Pós-Graduação, Ed. UFSCar: São Carlos, 1998.

5. Cagnin, M. A. H.; Quim. Nova 1993, 16,161.
6. Marchini, J. S.; Leite, J. P.; Velasco, I.T.; INFOCAPES 2001, 9, 5.

7. Pinto, A. C.; de Andrade, J. B.; Quim. Nova 1999, 22, 448.

8. Linard, P. M.; Coelho, P. M. Z., Costa, H. M. A.; Braz. J. Med. Res. 1996, 29, 555.

9. Ferreira, V. F.; Quim. Nova 2002, 25, 715

10. Scientific Citation Index, Journal Citation Reports 1998

11. Massie, B. M.; J. Card. Fail. 2002, 8, 363.

12. Kokko, H.; Trends Ecol. Evol. 1999, 14, 382.

13. Braga, M. M.; Azevedo, S. Em A Pós-Graduação no Brasil: A Formação e Trabalho de Mestre e Doutores no País Vol. 1; Velloso, J., ed.; CAPESUNESCO, Brasília, 2002.

14. Braga, M. M.; Azevedo, S.; Quim. Nova 2002, 25, 696.

15. de Andrade, J. B.; Schor, H. H. R.; do Nascimento, M. A. C.; Giordan, M.; Brocksom, T. J.; Aguilera, F. J. N.; Barreiro, E. J.; Quim. Nova 1995, 18, 97.

16. Brocksom, T. J.; de Andrade, J. B.; Quim. Nova 1997, 20, 29 e trabalhos citados.

17. da Gama, A. A. S.; Nome, F. J.; Machado, J. C.; Quim. Nova 1999, 22, 443.

18. Amin, M.; Mabe M.; Perspectives in Publishing 2000, 1, 1. 